\title{
Study of Histopathological Correlation of Breast Mass with Radiological and Cytological Findings.
}

\author{
Dr.A.Sarangan ${ }^{1}$,Prof.Dr.R.Geetha ${ }^{2}$,Dr.Sahaya Raj, Dr.B.Pushpa. \\ ${ }^{1}$ (Assistant professor, Department of pathology, kilpauk medical college, Chennai , India) \\ ${ }^{2}$ (Professor, Department of pathology, kilpauk medical college, Chennai, India)
}

\begin{abstract}
Breast cancer is the most common and remains the second leading cause of death in women. Triple assessment of breast mass includes clinical examination, imaging and fine needle aspiration cytology or core biopsy. Fine needle aspiration cytology and breast ultrasound is important in the characterization of breast lump to differentiate benign from malignant disease. All palpable breast masses require proper work up, early diagnosis and management.

Aim: 1. To correlate radiological and cytological findings of breast mass with histopathology studies.

2. To evaluate the role of cytology and sonomammography in diagnosing breast mass lesions.

Materials and methods: 50 patients with breast lumps were clinically examined, evaluated with Sonography and fine needle aspiration cytology in govt. kilpauk medical college, Chennai.. The lesions were assessed based on morphological criteria. Histopathological correlation was done, which was taken as standard. Sensitivity, specificity were derived for Sonography and cytology individually and also combined.

The main purpose of fine needle aspiration cytology in combination with radiological imaging of breast lumps is to confirm cancer preoperatively. Hence, correlation of Radiologic and cytologic findings is important for diagnosing and management of breast carcinoma and to avoid unnecessary surgery in benign conditions.
\end{abstract}

Keywords: Breast Cancer, Fnac, Ultrasound.

\section{Introduction}

Breast cancer is the most common cancer and leading cause of cancer deaths among women worldwide [1]. A breast lump is the most common symptom associated with both benign and malignant breast lesions . Therefore, a distinction of benign from malignant lump is of importance for patient and proper management.

Palpable breast masses, either self-detected or identified by clinical examination is common. Although most detected masses are benign, every woman presenting with breast mass should be evaluated to exclude or establish the diagnosis of cancer. Over 100,000 new breast cancer patients are diagnosed annually in India and according to WHO an estimated 70218 women $^{[2]}$ died due to breast cancer. Early detection of breast cancer in order to improve the cancer outcome and survival remains the keystone of breast cancer control.The established management of palpable breast masses includes triple assessment, which includes clinical examination, imaging and fine needle aspiration cytology .Fine needle aspiration cytology is a relatively simple, reliable, atraumatic, economical and complication free technique for the evaluation of mass lesions. Fine needle aspiration cytology of a breast lump is an accepted and established method to determine the nature of a breast lump. Sonomammography is the widely accepted and cost effective modality used for breast cancer screening in clinically suspected lesion. Though a definitive diagnosis is possible with imaging, for all the lesions histopathology study is proven essential for confirming the diagnosis. The present study was undertaken to evaluate the role of fine needle aspiration cytology and sonomammography in diagnosing breast mass lesions individually and when combined with histopathological correlation.

\section{II . Materials And Methods}

Present study is a prospective study conducted over a period of 5 months. A total of 50 patients with suspicious breast mass were evaluated with cytology and sonomammography. Clinical evaluation included the patient's demographic details, reproductive history, history of breast diseases, and physical examination. Ultrasound examination of the breast masses was done by an expert Sonologist in the department of radio diagnosis.The transducer was gently applied and both longitudinal and transverse scans were taken. The scans included information regarding the four features of the breast:(i) Shape Round/Oval or irregular. (ii) Margins Circumscribed or noncircumscribed. (iii) Width: AP ratio $>1.4$ or $=1.4$ (iv) Echogenicity Hyperechoic, Isoechoic or Hypoechoic. On the basis of these four features an impression about diagnosis was made. The studies were reviewed and reported according to BIRADS protocol . The radiologist's report was recorded as radiological diagnosis. Fine needle aspiration cytology was aseptically taken from the lesion using $23 \mathrm{G}$ needle and $5 \mathrm{ml}$ syringe. Cellular material was aspirated into a syringe and expelled onto slides. Four to six slides were prepared for each patient. A small or medium-sized drop of aspirate was put near the labelled end of a slide that 
was placed on a table. A second slide was used to spread the aspirated material along the specimen slide.All the smears were wet fixed in alcohol and stained with Hematoxylin and Eosin (H\&E).

Criteria for adequacy: There should be at least six clusters of ductal cells on each smear comprising 10 cells per cluster. At least two to three passes are made from the lump from all aspects.

Cytological categories for reporting of breast mass are described as follows.

1.Malignant

2.Suspicious

3.Atypical/indeterminate

4.Benign - specific diagnosis

5.Benign - non-specific

6.Unsatisfactory sample.

Criteria for malignancy: The morphological characteristics that were used to distinguish benign from malignant cells are as follows:

1. Abnormal grouping of cells

2. Decreased mutual adhesiveness

3. Changes in the nucleus

4. Increased/abnormal mitoses

5. Variation in size and shape of cells

6. Abnormal cytoplasmic inclusions .

Ultrasonography and Fine needle aspiration cytology diagnoses were respectively being recorded as benign, suspicious or malignancy. Suspicious cases were those, which fell in BIRADS IV category on mammography and showed atypical cells without frank malignancy on fine needle aspiration cytology. In this study, all suspicious cases were regarded as malignant. The confirmation of the ultrasound diagnosis and fine needle aspiration studies was made by histopathology which was done by an expert pathologist in the department of pathology.Informed consent was taken from the patients. Inclusion criteria were all patients with clinically palpable breast masses, strong family history- First degree relatives with breast carcinoma, operated case of breast carcinoma with contralateral breast mass. Exclusion criteria were age less than 35 years, very large and very tender breast, women with fungating mass and mass adherent to chest wall -where performing ultrasonography and cytology is difficult, very uncooperative patients,lactating and pregnant patients. Descriptive statistics were reported using mean and standard deviation for continuous variables, number and percentage for categorical variables. A correlation was done between cytology, sonomammography and histopathology results individually and combined. Sensitivity, specificity, positive predictive value, negative predictive value and accuracy were computed for each outcome. A p value less than 0.05 was considered statistically significant.

\section{Results}

In present study 106 cases were examined amongst which 50 cases were included, rest of the cases were normal or failed to follow-up and refused for consent. 50 cases were evaluated clinically and radiologically. Age of the patients ranged from 35 to 86 years with mean age of $49 \pm 4.2$ years. Cytologically there were 15 case of malignant and suspicious for malignant.Histopathologically, they were reported asductal carcinoma in situ, infiltrating ductal carcinoma, lobular and papillary carcinoma. There were total of 35 benign cases, fibroadenoma being the commonest followed by benign proliferative breast disease, fibrocystic disease, simple cysts, duct ectasia, mastitis, galactocele and least being phyllodes. On histopathological examination 1 benign case turned out to be malignant.

A case of malignant papillary carcinoma was misdiagnosed as proliferative breast disease without atypia. Out of 50 cases in our study 41 cases were detected as benign breast disease (BIRADS I and II) by ultrasound. Carcinoma of the breast was histologically found in 14 cases out of which 9 were correctly diagnosed by ultrasound, thus a sensitivity of $56.25 \%$. The sensitivity, specificity and accuracy of individual modality are derived based onTABLE -2,3,4,5. There was significant difference between cytology and ultrasonography in detecting breast disease ( $\mathrm{p}$ value $>0.05$ ). There is significant difference between individual modality and combined. 


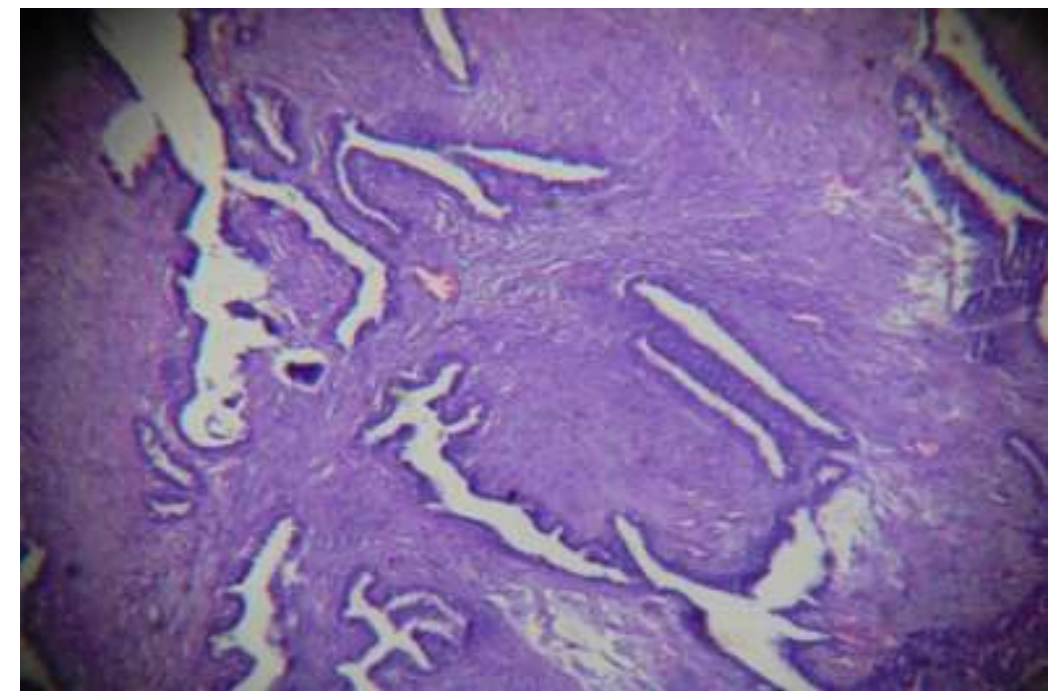

Fig-1 Section showing compressed ducts surrounded by stroma X 40(H\&E)- fibroadenoma.

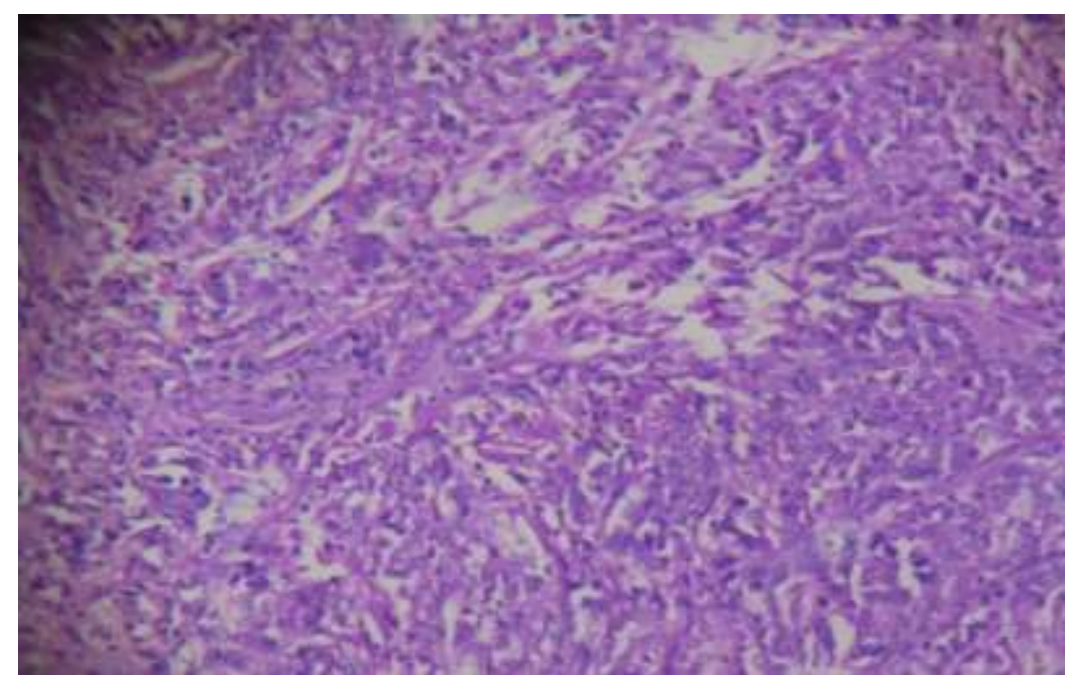

Fig-2- Section showing sheets of malignant pleomorphic hyperchromatic ductal epithelial cells X 40(H\&E) -infiltrating ductal carcinoma of breast.

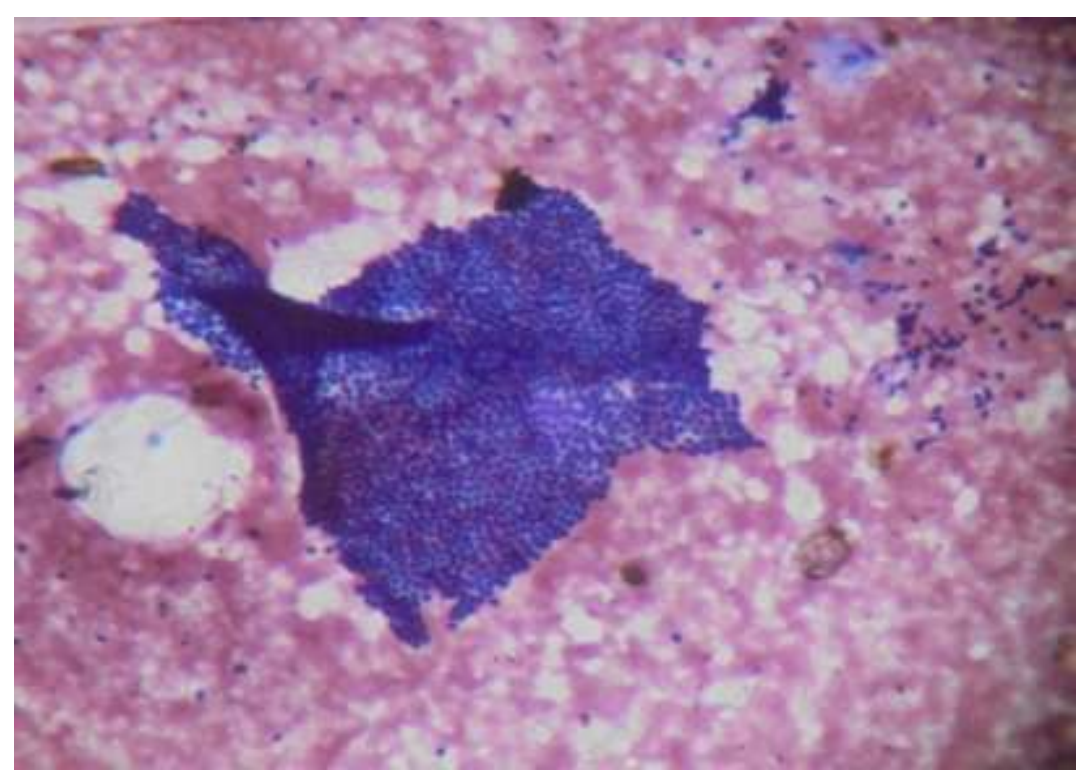

Fig-3- Smear showingmonolayered sheets of benign ductal epithelial cells X 10((H \&E)-Fibroadenoma. 


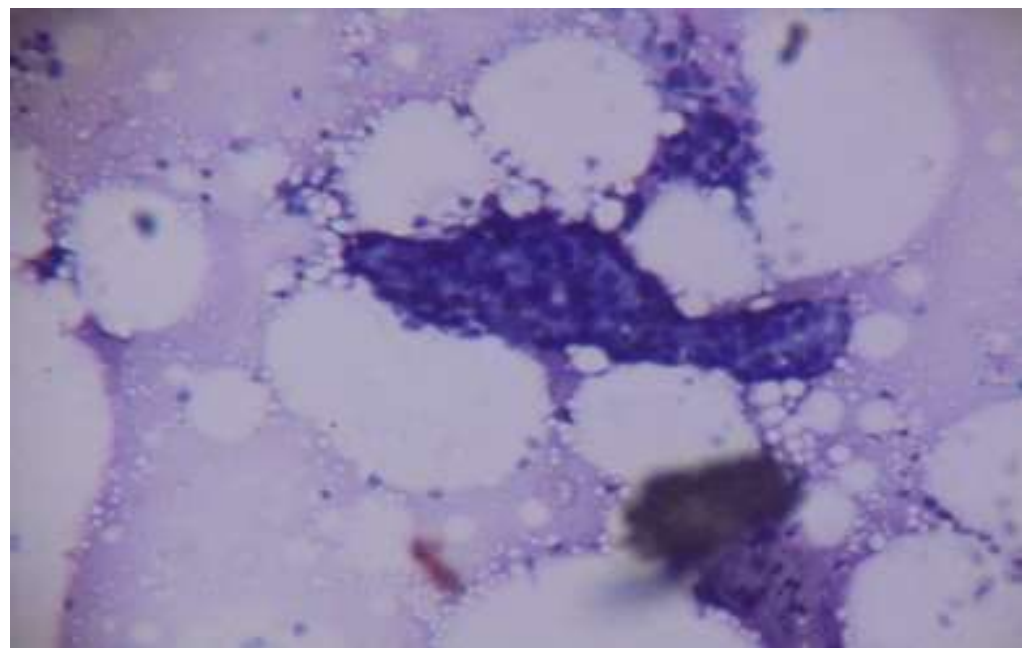

Fig-4 Smear showing hyperchromatic and pleomorphic epithelial cells X40(H\&E)-ductal carcinoma breast.

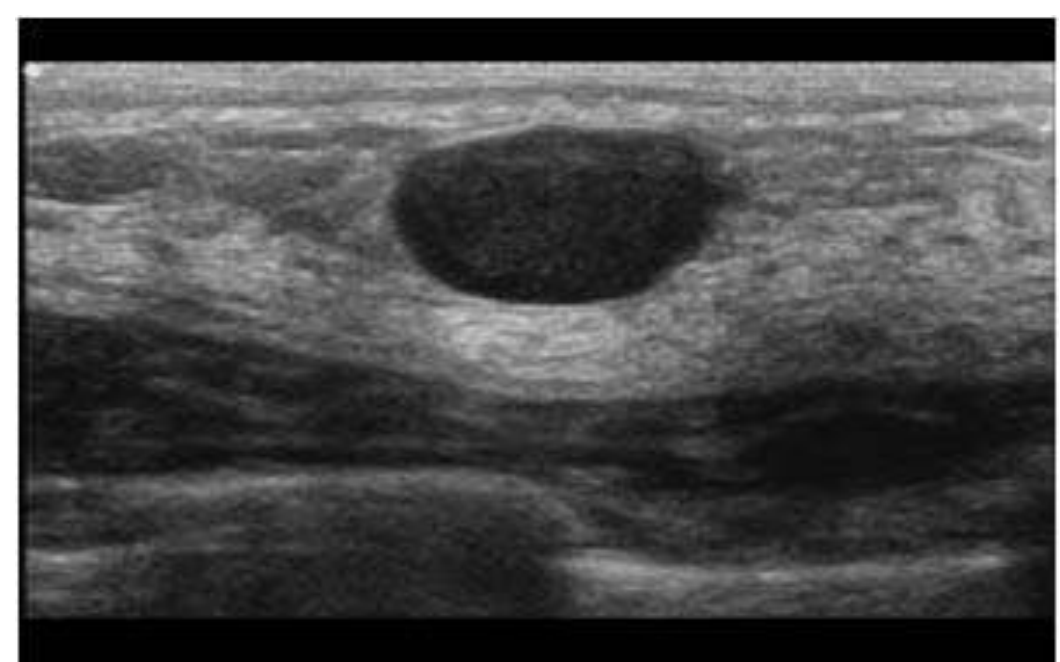

Fig-5Ultrasonogram showing well defined oval mass lesion with posterior enhancement (BIRADS II)

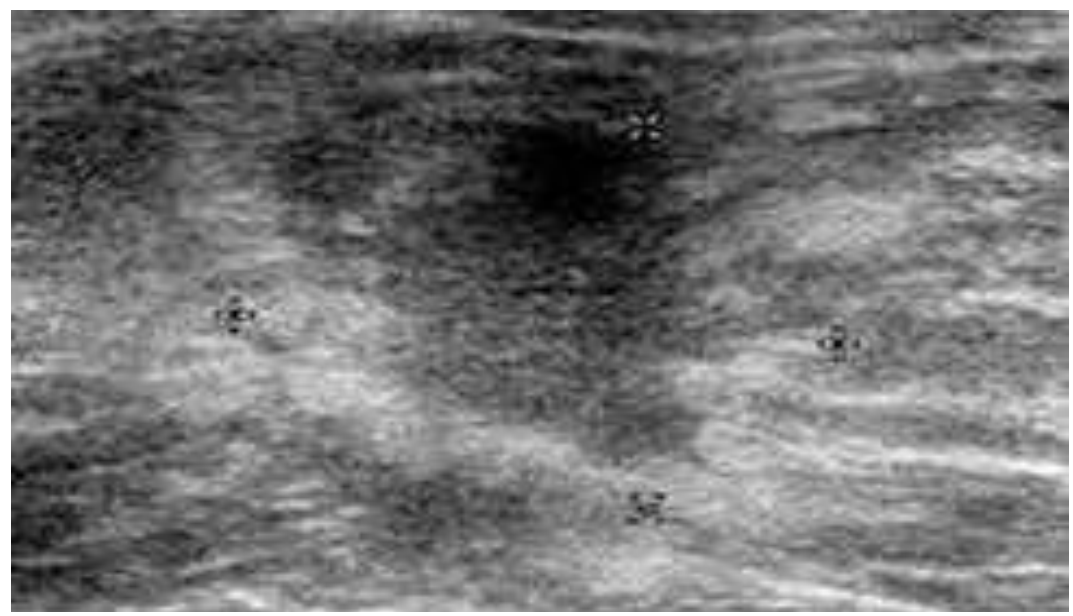

Fig-6Ultrasonogram showing hypoechoic mass with microlobulations\& irregular borders (BIRADS IV)

Table 1: Cytological Diagnoses of Breast diseases included in the study .

\begin{tabular}{|l|l|}
\hline Cytological Diagnosis & No. of Cases \\
\hline Fibroadenoma & 31 \\
\hline Benign Proliferative Disease & 2 \\
\hline Fibrocystic disease & 1 \\
\hline Phyllodes & 1 \\
\hline Malignancy & 15 \\
\hline
\end{tabular}


Table 2: Histopathological correlation VS Cytology

\begin{tabular}{|l|l|l|}
\hline Breast Lesion & Cytology & Histopathology \\
\hline Fibroadenoma & 31 & 31 \\
\hline Fibrocystic disease & 1 & 1 \\
\hline Benign Proliferative Breast Disease & 2 & 1 \\
\hline Phyllodes tumour & 1 & 1 \\
\hline Papillary carcinoma & 0 & 1 \\
\hline Infiltrating Ductal carcinoma & 15 & 15 \\
\hline
\end{tabular}

Table -3. Cytology vs histopathology in differentiation between benign and malignant breast mass.

\begin{tabular}{|l|l|l|l|l|}
\hline \multirow{2}{*}{} & \multicolumn{2}{|c|}{ Cytology } & \multicolumn{2}{c|}{ Histopathology } \\
\cline { 2 - 5 } & No. & Percentage & No. & Percentage \\
\hline Benign & 35 & $70 \%$ & 34 & $68 \%$ \\
\hline Malignant & 15 & $30 \%$ & 16 & $32 \%$ \\
\hline
\end{tabular}

Sensitivity $=\underline{\text { True Positive }} \times 100=93.75 \%$

True Positive + False negative.

Specificity $=\underline{\text { True negative }} \times 100=100 \%$

True Negative + False Positive.

Accuracy $=\quad$ True Positive + True Negative $\times 100=98.03 \%$

True Positive + False Positive + True Negative + False Negative.

Positive Predictive Value $=$ True Positive $\times 100=100 \%$

True Positive + False Positive.

Negative Predictive Value $=\underline{\text { True Negative }} \times 100=97.22 \%$

True Negative + False Negative

Table 4. Categories Of Breast Lesions on Cytology And Ultrasonography.

\begin{tabular}{|l|l|l|l|l|l|}
\hline Benign BIRADS & No. of cases & Percentage & Benign Cytology & No. Of Cases & Percentage \\
\hline II & 35 & $70 \%$ & Benign & 29 & $58 \%$ \\
\hline III & 6 & $12 \%$ & $\begin{array}{l}\text { Benign -non } \\
\text { specific }\end{array}$ & 6 & $12 \%$ \\
\hline $\begin{array}{l}\text { Malignant } \\
\text { BIRADS }\end{array}$ & & Malignant cytology & & \\
\hline IV & 6 & $12 \%$ & Suspicious/atypical & 4 & $8 \%$ \\
\hline V & 3 & $6 \%$ & malignancy & 11 & $22 \%$ \\
\hline
\end{tabular}

Table-5.Ultra SonographyVs Histopathology in differentiation between benign and malignant breast mass.

\begin{tabular}{|l|l|l|l|l|}
\hline \multirow{2}{*}{} & \multicolumn{2}{|l|}{ Ultrasonograpy } & \multicolumn{2}{c|}{ Histopathology } \\
\cline { 2 - 5 } & No. & Percentage & No. & Percentage \\
\hline Benign & 41 & $82 \%$ & 34 & $68 \%$ \\
\hline Malignant & 9 & $18 \%$ & 16 & $32 \%$ \\
\hline
\end{tabular}

Sensitivity $=\underline{\text { True Positive }} \times 100=56.25 \%$

True Positive + False negative.

Specificity $=\underline{\text { True negative }} \times 100=82.92 \%$

True Negative + False Positive.

Accuracy $=$ True Positive + True Negative $\times 100=75.43 \%$

True Positive + False Positive + True Negative + False Negative.

Positive Predictive Value $=\underline{\text { True Positive }} \times 100=56.25 \%$

True Positive + False Positive

Negative Predictive Value $=\underline{\text { True Negative }} \quad \times 100=82.92 \%$

True Negative + False Negative.

Table-6.Ultra Sonography Vs Cytology in differentiation between benign and malignant breast mass.

\begin{tabular}{|c|l|l|l|l|l|l|l|}
\hline \multirow{2}{*}{} & \multicolumn{2}{|l|}{ Ultrasonograpy } & \multicolumn{2}{|l}{ Cytology } & \multicolumn{2}{|l}{ P value } \\
\cline { 2 - 8 } & No. & Percentage & No. & Percentage & No. & percentage & \\
\hline Benign & 41 & $82 \%$ & 35 & $68 \%$ & 34 & 68 & \multirow{2}{*}{ P $>0.05$} \\
\hline Malignant & 9 & $18 \%$ & 15 & $32 \%$ & 16 & 32 & \\
\hline
\end{tabular}




\section{Discussion}

Breast cancer is one of the most prevalent cancers in the world among women. FNAC of breast lumps is an accepted and established method for determining the natures of breast lumps with a high degree of accuracy. Application of Fine Needle Aspiration for the diagnosis of palpable breast masses was first introduced by Martin and Ellis in $1930^{[3]}$. Ultrasound is also useful in guiding FNAC or biopsies and more reliable in evaluation of dense breasts. The purpose of this present study was to determine the value of fine needle aspiration cytology and ultrasonography in the diagnosis of breast carcinoma and to compare the result of FNAC and Ultra sonography with histological diagnosis to assess its accuracy. Early screening and diagnosis of breast lesions and categorization into different groups of breast pathology can be helpful in accurate management of the breast lesions. SonoMammography is an essential component in the pre-operative assessment of breast cancer. It serves to characterize and determine the extent of the mass and to evaluate the breasts for clinically occult lesions. Overall, sensitivity of Ultrasonogram was $56.25 \%$ with specificity of $82.92 \%$. Sensitivity of ultrasonography in detecting benign lesions were high because small cysts and fibroadenomas are better seen even in dense breasts and Ultrasonogram differentiates cyst from solid lesions. These results correlate with other studies Kailash Singh et al ${ }^{[4]}$ found that the sensitivity of ultrasound in detection of palpable breast lumps was $95 \%$. The sensitivity, specificity and positive predictive value of ultrasound in fibroadenoma of the breast was $81.6 \%, 94.7 \%$ and $91.2 \%$ respectively. In another study of Wasan et $\mathrm{al}^{[5]}$ with $100 \%$ sensitivity and $91.6 \%$ specificity, its positive predictive value was as high as $57.1 \% \&$ the negative predictive value was $100 \%$. Benign lesions of the breast were more readily diagnosed by ultrasound than malignant lesions.According toNandan Kumar et $\mathrm{al}^{[6]}$ the sensitivity and specificity in detecting breast lump is 85.45 and 89.31 , these values are much higher compared to our study.Fine needle aspiration cytology of breast lump is an accepted and established method to determine the nature of the lump and it may play an important role when it is difficult to determine the nature of breast lump by clinical examination. The study of Puja B. Jarwani et $\mathrm{al}^{[7]}$, Ambedkar Raj KulandaiVelu et $\mathrm{al}^{[8]}$ has been found to have sensitivity ranging from $82 \%$ to $97.5 \%$ and specificity of more than $99 \%$.

In this study, sensitivity, specificity and accuracy of breast FNAC in diagnosis of malignancy was 93.5\%,100\% and 98.3\% respectively. The results of Dr manju et al ${ }^{[9]}$; Shanmugasamy K et al ${ }^{[10]}$ were quite comparable to the findings of our studies. In the present study, False positive results were very minimal compared to False negative results.False negative and inconclusive reports were commonly resulted from hemorrhagic aspirates from highly vascular tumors, acellular aspirates from very hard lumps, and aspirates from cystic lumps and sampling error. This can be minimized by proper localization and aspiration technique ${ }^{[11]}$. Proper localization can be aided using ultrasound. According to Nicholson et al,the skill and experience of the pathologist in obtaining the specimen and interpretation can reduce false negative as much as possible ${ }^{[12]}$. The results of our study showed FNAC of breast lump to be a reliable method to diagnose breast lump with high accuracy. Triple assessment by clinical, radiological and FNAC can produce $99 \%$ accuracy for both benign and malignant lesions as false negative results can mislead a clinician and cause a delay in appropriate investigation, diagnoses, and treatment. Furthermore, it needs absolute collaboration between surgeons, radiologist and pathologist. The triple assessment is therefore cost effective, easy to perform and time saving approach, however, it can be applied only in those institutions where excellent imaging facilities as well as services of a cytopathologist are available.

\section{Conclusion}

Present study confirms the higher combined sensitivity, specificity and accuracy for ultrasonography and fine needle aspiration cytology for detection of breast masses including malignancies. USG is better in cystic lesion ectasia, inflammatory lesion and dense breast evaluation, whereas cytology is better in detection of malignancy. The triple assessment is an accurate, simple and cost-effective method for the evaluation of breast cancer and can be applied at our centre as a safe alternative for open biopsy when it is concordant and open biopsy should be reserved only for disconcordant results. This will subsequently reduce the number of openbreast biopsies significantly.

\section{References}

[1]. Abdullah, P., Malik, A., Zahir, N., Zahur-ur-Rehman, Abdus S. \&Mehmood A.(1999) Breast lumps-what they actually represent. Journal of College of Physicians and SurgeonsPakistan 9, 46-48.

[2]. Rashmi Bawa et al. Breast Cancer In India. Int J Med Res Prof.2015;1(2);27-31.

[3]. Martin HE, Ellis EB. Biopsy by needle puncture and aspiration. Ann Surg 1930;92:169-18.

[4]. Kailash Singh, Tariq Azad, Ghanshyam Dev Gupta et al .The Accuracy of Ultrasound in Diagnosis of Palpable Breast Lumps. JK Science. 2008;10:4.

[5]. Wasan et al: Non Palpable Breast Mass.Mustansiriya Medical Journal Volume 15 Issue 1 April 201.

[6]. Nandan Kumar1, Shilpa N2 et al .Mammographic And Sonomammographic Evaluation Of Breast Masses With Pathological Correlation .Innovative Journal of Medical and Health Science 6:5 September - October (2016) 132 - 135. 
[7]. Puja B. Jarwani, Daxita C. Patel, Shantibhai M. Patel, AnupamaDayal..Fine Needle AspirationCytology in A Palpable Breast. GCSMC J Med Sci Vol (II) No (II) July-December 2013.

[8]. Ambedkar Raj KulandaiVelu, Banushree C Srinivasamurthy, Jhansi Rani.Cytological evaluation of benign breast lesions with histopathological correlation.Indian Journal of Pathology and Oncology, January - March 2016;3(1);7-10.

[9]. Dr.Manju.T.Vala, Dr.Alpesh. Goswami, Dr.S.K.Suri .Comparative study of cytological and histopathological finding in breast lesion .IOSR Journal of Dental and Medical Sciences (IOSR-JDMS) e-ISSN: 2279-0853, p-ISSN: 2279-0861.Volume 13, Issue 7 Ver. III (July. 2014), PP 05-07.

[10]. Shanmugasamy K, AnandrajVaithy $\mathrm{K}$ et al .Cytolo - histological correlation of breast lump - as a part of internal quality. Indian Journal of Pathology and Oncology, April-June 2016;3(2);328-335..

[11]. McManus, DT. \& Anderson, N.H. (2001) Fine needle aspiration cytology of the breast. Current DiagnosticPathology7,262-271.

[12]. Nicholson,S.,Sainsbury,J.R.C., Wadehra,V.\&Needham G.K. (1988)Use of FNAC withimmediate reporting in the diagnosis ofbreast disease. British Journal of Surgery75, 847-850. 